Aim of the study: Interleukin (IL)-17 and IL-23 play roles in inflammation and autoimmunity. The function of the IL-17/IL-23 pathway has not been completely evaluated in cancer patients. We aimed to investigate serum IL-17 and IL-23 levels and their relationship with clinicopathological and biochemical parameters in lung cancer patients.

Material and methods: Forty-five lung cancer patients and 46 healthy volunteers were included in the study. IL-17 and IL-23 measurements were made with the ELISA method. The ages of patients (53-84 years) and healthy subjects (42-82 years) were similar.

Results: Serum IL-23 levels were higher in lung cancer patients than in healthy subjects (491.27 \pm 1263.38 $\mathrm{pg} / \mathrm{ml}$ vs. $240.51 \pm 233.18 \mathrm{pg} / \mathrm{ml}$; $p=0.032$ ). IL-23 values were higher in small cell lung cancer (SCLC) patients than in non-small cell lung cancer (NSCLC) patients (1325.30 \pm 2478.06 $\mathrm{pg} / \mathrm{ml}$ vs. $229.15 \pm 103.22 \mathrm{pg} / \mathrm{ml}$ $p=0.043)$. Serum IL-17 levels were lower in the patients, but the difference was not statistically significant $(135.94 \pm 52.36 \mathrm{pg} / \mathrm{ml}$ vs. 171.33 $\pm 133.51 \mathrm{pg} / \mathrm{ml} ; p=0.124)$. Presence of comorbid disease (diabetes mellitus, hypertension or chronic obstructive lung disease) did not have any effect on the levels of IL-17 or IL-23. Erythrocyte sedimentation rate values were positively correlated with cytokine levels, but serum albumin levels were negatively correlated.

Conclusions: Serum IL-23 levels are elevated in lung cancer patients, particularly those with SCLC. IL-17 and IL-23 values are correlated with inflammatory markers in the patients.

Key words: IL-17, IL-23, lung cancer, Th17 cells.

Contemp Oncol (Pozn) 2016; 20 (3): 215-219 DOI: $10.5114 /$ wo.2016.61562

\section{The inflammatory cytokine interleukin-23 is elevated in lung cancer, particularly small cell type}

\author{
Caner Cam ${ }^{1}$, Bulent Karagoz ${ }^{2}$, Tuba Muftuoglu ${ }^{3}$, Oguz Bigi ${ }^{2}$, Levent Emirzeoglu², \\ Serkan Celik², Alpaslan Ozgun², Tolga Tuncel², Cihan Top ${ }^{4}$
}

${ }^{1}$ Department of Internal Medicine, Kayseri Military Hospital, Istanbul, Turkey ${ }^{2}$ Department of Medical Oncology, GATA Haydarpasa Hospital, Istanbul, Turkey ${ }^{3}$ Department of Biochemistry, GATA Haydarpasa Hospital, Istanbul, Turkey ${ }^{4}$ Department of Internal Medicine, GATA Haydarpasa Hospital, Istanbul, Turkey

\section{Introduction}

Lung cancer is one of the most common cancers in the world. It has the highest mortality rate. According to the etiological factors such as genetic modification, treatment has been individualized in lung cancer [1]. Thus explanation of etiologic factors is important. Besides smoking, many genetic and environmental factors also play a role in the etiology of lung cancer.

Inflammation is associated with several malignancies. It may be related to the initiation, promotion, progression, surveillance, and control of the malignant process [2]. Chronic inflammation may create structural, genetic, and epigenetic alterations in tumor initiation. Moreover, tumor-associated inflammation promotes tumor growth and metastatic formation. Inflammatory markers such as C-reactive protein (CRP) and high-density lipoprotein cholesterol (HDL cholesterol) have been altered and have prognostic value in lung cancer patients [3, 4].

CD4+ T helper (Th) cells are mediators of the cellular immune response. Th cells were first referred to as Th1 and Th2 due to cytokine expression patterns. Then Th17 cells, a subset of CD4+ T cells, were described. These cells produce IL-17, IL-21, and IL-22 and play a role in inflammation and autoimmunity [5]. IL-17 secretion is regulated by several factors, such as IL-23. IL-23 is secreted in dendritic cells and mononuclear macrophages [6]. These inflammatory cytokines, IL-23 and IL-17, were found to play important pathogenic roles in several inflammation related carcinomas. IL-23 and IL-23 receptors have been found elevated and IL-17A has been linked to an adverse prognostic outcome and rapid progression to metastatic disease in colorectal carcinomas. In animal models of colorectal carcinogenesis inhibition of these cytokines attenuates tumor development and malignant progression [6].

Although IL-17 and IL-23 play roles in several biological processes, the function of the IL-17/IL-23 pathway has not been completely evaluated in lung cancer patients. We investigated serum IL-17 and IL-23 levels and their relationship with clinicopathological and biochemical parameters in lung cancer patients.

\section{Material and methods}

Forty-six lung cancer patients and 45 control subjects were included in the study. The patients were treated in a medical oncology department; the healthy subjects were referred from check-up and chest medicine clinics and did not have any pathologic condition except chronic obstructive pulmonary disease (COPD), hypertension (HT), or diabetes mellitus (DM). Written informed consent was obtained from all participants. This study was approved 
by the local ethical committee in December 2012. Demographic and clinical data were collected from clinical documentation.

Patients with chronic inflammatory diseases (except COPD, HT, or DM), rheumatic diseases, or primary immune deficiency, and those who used immunosuppressive medications were not included in the study.

\section{Enzyme-linked immunosorbant assay (ELISA) and biochemical analyses}

All biochemical investigations were performed under the same conditions in the Laboratory of Biochemistry, GATA Haydarpasa Hospital. The serum samples were centrifuged at 5000 RPM for 5 minutes. The supernatants were divided and collected at $-80^{\circ} \mathrm{C}$ until the test day. The following parameters were determined:

- IL-17 was determined using Human IL-17 ELISA Kit (BOSTER Immunoleader No: EK0430, Boster Biological Technology Co., Ltd, 40459, Fremont, CA 94538, USA). Boster's human IL-17 ELISA Kit was based on standard sandwich enzyme-linked immune-sorbent assay technology. The measurement range of the assay is 31.2$2000 \mathrm{pg} / \mathrm{ml}$ and sensitivity is $<1 \mathrm{pg} / \mathrm{ml}$;

- IL-23 was determined using the Human IL-23 ELISA Kit (OmniKine No: OK-0243, Assay Biotechnology Company, CA 94089, USA). The measurement range of the assay is $63-8000 \mathrm{pg} / \mathrm{ml}$ and sensitivity is $<25 \mathrm{pg} / \mathrm{ml}$.

The measurements were performed according to the manufacturer's protocol. At the end readings were made directly at $450 \mathrm{~nm}$ with a microplate reader (DAR800, Diagnostic Automation, CA 91302, USA). The results were expressed as pg/ml.

\section{Statistics}

SPSS software was used for statistical analysis. All data are given as mean \pm standard error of the mean (SEM).
Normal distributions of group parameters were described using the Kolmogorov-Smirnov test. The Mann-Whitney rank sum test was used to compare differences between groups. Fisher's exact was used for categorical variables. Correlation between groups was calculated using Pearson's correlation test. Statistical significance was accepted if $p<0.05$.

\section{Results}

Forty-six lung cancer patients and 45 controls were included in the study. Among patients, 35 had non-small cell lung cancer (NSCLC) (18 squamous cell carcinoma, 15 adenocarcinoma, 1 adenosquamous carcinoma, and 1 NSCLC, not otherwise specified), and 11 had small cell lung cancer (SCLC). Eight SCLC patients had extensive stage, and 3 had limited disease. Fourteen NSCLC patients were metastatic (11 stage III, 5 stage II, and 5 stage I). Lung cancer group and control groups were not different with regard to age, sex, smoking status, or comorbidities (Table 1).

In biochemical parameters, acute-phase reactants (erythrocyte sedimentation rate [ESR], C-reactive protein [CRP], ferritin, and serum albumin level) were different in lung cancer patients than in controls (data not shown). Leukocyte count, platelet count, neutrophil count, monocyte count, plateletcrit, urea, aspartate aminotransferase (AST), alanine aminotransferase (ALT), and gamma glutamyl transpeptidase (GGT) were elevated; creatinine kinase, HDL cholesterol, serum sodium level, hemoglobin, and hematocrit were lower in lung cancer patients.

Serum IL-17 levels were lower in lung cancer patients than in controls, but the difference was not statistically significant. However, serum IL-23 level was higher in patients than in controls (Table 2). Among lung cancer patients, serum IL-23 levels of SCLC patients were higher than in NSCLC patients. Serum IL-17 levels were similar in both NSCLC and SCLC patients (Table 2). In lung cancer

Table 1. Demographics of lung cancer patients and controls

\begin{tabular}{|c|c|c|c|c|c|}
\hline \multirow[t]{2}{*}{ Parameter } & \multicolumn{2}{|c|}{ Lung cancer patients } & \multicolumn{2}{|c|}{ Control subjects } & \multirow[t]{2}{*}{$p$} \\
\hline & $n$ & $\%$ & $n$ & $\%$ & \\
\hline \multicolumn{6}{|l|}{ Sex } \\
\hline Male & 41 & 89.1 & 35 & 77.78 & 0.17 \\
\hline Female & 5 & 10.9 & 10 & 22.22 & \\
\hline \multicolumn{6}{|l|}{ Comorbidity } \\
\hline COPD & 8 & 17.4 & 12 & 26.7 & 0.32 \\
\hline Hypertension & 21 & 45.7 & 20 & 44.4 & 1.00 \\
\hline Diabetes mellitus & 9 & 19.6 & 3 & 6.7 & 0.12 \\
\hline \multicolumn{6}{|l|}{ Smoking } \\
\hline Smoker & 27 & 58.7 & 21 & 46.7 & 0.08 \\
\hline Non-smoker & 10 & 21.7 & 18 & 40 & \\
\hline Ex-smoker & 9 & 19.6 & 6 & 13.3 & \\
\hline $\begin{array}{l}\text { Ages } \\
\text { (range) }\end{array}$ & \multicolumn{2}{|c|}{$(42-82)$} & \multicolumn{2}{|c|}{$(53-84)$} & 0.10 \\
\hline
\end{tabular}


Table 2. Serum IL-17 and IL-23 levels in lung cancer patients and controls

\begin{tabular}{|c|c|c|c|c|}
\hline \multirow[t]{2}{*}{ Parameter } & \multicolumn{2}{|c|}{ IL-17 } & \multicolumn{2}{|c|}{ IL-23 } \\
\hline & & $p^{*}$ & & $p^{*}$ \\
\hline \multicolumn{5}{|l|}{ Groups } \\
\hline Lung cancer patients & $135.94 \pm 52.36$ & & $491.27 \pm 1263.38$ & \\
\hline Control subjets & $171.33 \pm 133.51$ & 0.124 & $240.51 \pm 233.18$ & 0.032 \\
\hline \multicolumn{5}{|l|}{ Lung cancer patients } \\
\hline $\mathrm{SCLC}$ & $144.89 \pm 57.81$ & & $1325.3 \pm 2478.06$ & \\
\hline NSCLC & $133.13 \pm 51.11$ & 0.542 & $229.15 \pm 103.22$ & 0.043 \\
\hline \multicolumn{5}{|l|}{ NSCLC } \\
\hline Non-metastatic & $136.84 \pm 50.38$ & & $246.34 \pm 98.13$ & \\
\hline Metastatic & $127.57 \pm 53.59$ & 0.727 & $203.37 \pm 108.88$ & 0,235 \\
\hline
\end{tabular}

SCLC - small cell lung cancer; NSCLC - non-small cell lung cancer

*Mann-Whitney $U$ test

patients, serum IL-17 levels were not different between hypertensive and normotensive, diabetic and non-diabetic, or COPD and non-COPD patients. IL-23 levels were lower in diabetic and COPD patients than in non-diabetic and nonCOPD patients, but the difference was not statistically significant in this small subpopulation of the study (Table 3). Levels of these interleukins were similar in metastatic and non-metastatic NSCLC patients and not different among patients of different sexes (Table 2).

ESR had a positive correlation with IL-17 and IL-23 levels (respectively $r=+0.525 ; p=0.002$ and $r:+0.399 ; p=$ $0.021)$. A negative correlation was detected between serum albumin and IL-17 level $(r=-0.418 / p=0.004)$, but not IL-23. High serum creatinine level was associated with high serum IL-23 level $(r=+0.294 / p=0.047)$ (not IL-17). Age, serum ferritin level, CRP or other biochemical parameters did not correlate with serum interleukin levels.

\section{Discussion}

In this study, we investigated two inflammatory cytokines, IL-17 and IL-23, in peripheral blood from lung cancer patients. We found that serum IL-23 level is elevated in lung cancer, particularly SCLC patients, but not IL-17. Both interleukins correlate with some inflammatory markers (e.g. ESR, albumin). This study has some limitations. First, the study population was small, so subgroup or survival analyses could not be performed. Second, Th17 cells and regulatory $T$ (Treg) cell counts were not studied. To the best of our knowledge, this study is the first to investigate serum IL-17 and IL-23 levels together in lung cancer patients.

The relationship between IL-17 and cancer has already been established [7]. In the initial stage of tumorigenesis, increasing IL-17 presence has been demonstrated in the tumor microenvironment [8]. IL-17 is released as a response to the secretion of lactate via IL-23 dependent and independent pathways [9]. The increase in IL-17 can trigger the release of IL- 6 and activation of the STAT3 pathway and NF- $\mathrm{B}[10,11]$. Beside the tumor microenvironment,
IL-17 has also been found at a high level in malignant pleural effusion [12, 13].

There are also studies about whether IL-17 level is a serum marker in cancer patients. In lung cancer patients, some studies have reported that serum IL-17 is high and a prognostic marker compared to healthy subjects [14, 15], but some showed that it is not different [15]. Xu et al. [14] investigated the baseline serum level of IL-17 in NS$\mathrm{CLC}$ patients. They found that serum IL-17 level is higher in patients than healthy subjects and high serum IL-17 concentration correlates with shorter survival and presence of metastasis. Li et al. [15] also observed an increased IL-17 level and increased TH17 cell and Treg cell counts in NSCLC patients. However, Brusino et al. [16] reported that serum IL-17 level was similar in lung cancer patients as in healthy volunteers, although its level was higher in exhaled breath condensate. In addition, Teng et al. stated that despite the clear link between IL-23 and Th17 cells, the role of IL-17 in tumor development has not been fully explained [17]. In our present study, we were unable to show that serum IL-17 level is different in lung cancer patients, although we found increasing Treg cells in a previous study [18] and immunosuppressive HLA-G expression (gastric cancer) [19].

Interleukin 23, another study cytokine, supports Th17 cell propagation, whose development is induced by IL-6 and TGF- $\beta$ [20]. IL-17 plays a role together with IL-23 in several biologic processes; thus it is named the IL-23/IL-17 pathway. Among these biologic processes, the relationship of cancer with IL-23, like IL-17, has been investigated in previous studies. Interleukin 23 is also elevated in the tumor microenvironment [21,22] and is associated with poor prognosis [23]. It was also found that IL-23 was elevated in serum from cancer patients. Ljujic et al. [24] observed a higher serum IL-23 level and its correlation with tissue expression of vascular endothelial growth factor (VEGF) in colorectal cancer (CRC) patients. Adamo et al. [25] reported an increased serum IL-23 level in resected CRC and post-chemotherapy CRC patients compared to healthy subjects. Stanilov et al. [26] investigated the association 
Table 3. Serum IL-17 and IL-23 levels and comorbidities in lung cancer patients

\begin{tabular}{|c|c|c|c|c|}
\hline \multirow[t]{2}{*}{ Parameter } & \multirow[t]{2}{*}{ IL-17 } & \multicolumn{3}{|c|}{ IL-23 } \\
\hline & & $p$ & & $p$ \\
\hline \multicolumn{5}{|l|}{ Hypertension } \\
\hline Hypertensive & $143.04 \pm 38.36$ & & $424.76 \pm 853.3$ & \\
\hline Normotensive & $129.98 \pm 61.92$ & 0.201 & $547.14 \pm 1542.36$ & 0.877 \\
\hline \multicolumn{5}{|l|}{ Diabetes Mellitus } \\
\hline Diabetic & $131.66 \pm 55.75$ & & $267.11 \pm 134.08$ & \\
\hline Non-diabetic & $136.98 \pm 52.26$ & 0.935 & $545.8 \pm 1405.55$ & 0.765 \\
\hline \multicolumn{5}{|l|}{ COPD } \\
\hline COPD patients & $110.87 \pm 39.19$ & & $248,14 \pm 106.37$ & \\
\hline Non-COPD & $141.22 \pm 53.66$ & 0.201 & $542,46 \pm 1386.95$ & 0.898 \\
\hline
\end{tabular}

* Mann-Whitney $U$ test

of serum levels of IL-23 and IL-12p40 with survival of patients with CRC. Interleukin 23 was not a prognostic marker. Gangemi et al. [27] found that IL-23 level was higher in breast cancer patients than controls. They claimed that a higher IL-23 value has negative prognostic significance. Besides these cancer studies, IL-23 levels have not been investigated in serum from lung cancer patients. In our study, we have shown for the first time that serum IL-23 is elevated in lung cancer patients compared to healthy subjects. Interleukin 23 level is increased more in SCLC than NSCLC patients.

In conclusion, serum IL-23 level is elevated in lung cancer, particularly SCLC patients, but not IL-17. While the prognostic value of the IL-17/IL-23 pathway was not addressed in this study, further studies including more cases are required.

The authors declare no conflict of interest.

\section{References}

1. Karagoz B, Bilgi O. Individualized systemic treatment of lung cancer. Dis Mol Med 2013; 1: 15-8.

2. Dvorak HF. Tumors: wounds that do not heal. Similarities between tumor stroma generation and wound healing. N Engl J Med 1986; 315: 1650-9.

3. Ni XF, Wu P, Wu CP, Ji M, Wu J, Gu XF, Jiang ZX. Elevated serum C-reactive protein, carcinoembryonic antigen and N2 disease are poor prognostic indicators in non-small cell lung cancer. Asia Pac J Clin Oncol 2015; 11: e22-e30.

4. Chi PD, Liu W, Chen H, Zhang JP, Lin Y, Zheng X, Liu W, Dai S. High-density lipoprotein cholesterol is a favorable prognostic factor and negatively correlated with C-reactive protein level in nonsmall cell lung carcinoma. PLoS One 2014; 9: e91080.

5. Korn T, Bettelli E, Oukka M, Kuchroo VK: IL-17 and Th17 Cells. Annu Rev Immunol 2009; 27: 485-517.

6. Wang K, Karin M. The IL-23 to IL-17 cascade inflammation-related cancers. Clin Exp Rheumatol 2015; 33 (Suppl): S87-90.

7. Yang B, Kang H, Fung A, Zhao H, Wang T, Ma D. The role of interleukin 17 in tumour proliferation, angiogenesis, and metastasis. Mediators Inflamm 2014; 2014: 623759.

8. Murugaiyan G, Saha B: Protumor vs antitumor functions of IL-17. J Immunol 2009; 183: 4169-75.
9. Yabu M, Shime H, Hara H, Saito T, Matsumoto M, Seya T, Akazawa T, Inoue N. IL-23-dependent and -independent enhancement pathways of IL-17A production by lactic acid. Int Immunol 2011; 23: 29-41.

10. Wang L, Yi T, Kortylewski M, Pardoll DM, Zeng D, Yu H. IL-17 can promote tumor growth through an IL-6-Stat3 signaling pathway. J Exp Med 2009; 206: 1457-64.

11. Yu H, Pardoll D, Jove R. STATs in cancer inflammation and immunity: a leading role for STAT3. Nat Rev Cancer 2009; 9: 798-809.

12. Xu CH, Zhan P, Yu LK, Zhang XW. Diagnostic value of pleural interleukin 17 and carcinoembryonic antigen in lung cancer patients with malignant pleural effusions. Tumour Biol 2014; 35: 1599-603.

13. Klimatsidas M, Anastasiadis K, Foroulis C, Tossios P, Bisiklis A, Papakonstantinou C, Rammos K. Elevated levels of anti inflammatory IL-10 and pro inflammatory IL-17 in malignant pleural effusions. J Cardiothorac Surg 2012; 7: 104.

14. Xu C, Hao K, Yu L, Zhang X. Serum interleukin-17 as a diagnostic and prognostic marker for non-small cell lung cancer. Biomarkers 2014; 19: 287-90.

15. Li S, Li Y, Qu X, Liu X, Liang J. Detection and significance of TregFoxP3(+) and Th17 cells in peripheral blood of non-small cell lung cancer patients. Arch Med Sci 2014; 10: 232-9.

16. Brussino L, Culla B, Bucca C, et al. Inflammatory cytokines and VEGF measured in exhaled breath condensate are correlated with tumor mass in non-small cell lung cancer. J Breath Res 2014; 8: 027110.

17. Teng MW, Andrews DM, McLaughlin N, et al. IL-23 suppresses innate immune response independently of IL-17A during carcinogenesis and metastasis. Proc Natl Acad Sci U S A 2010; 107: 8328-33.

18. Karagoz B, Bilgi O, Gumus M, et al. CD8+CD28- cells and CD4+CD25+ regulatory $T$ cells in the peripheral blood of advanced stage lung cancer patients. Med Oncol 2010; 27: 29-33.

19. Tuncel T, Karagoz B, Haholu A, Ozgun A, Emirzeoglu L, Bilgi O, Kandemir EG. Immunoregulatory function of HLA-G in gastric cancer. Asian Pac J Cancer Prev 2013; 14: 7681-4.

20. Chen Z, O'Shea JJ. Th17 cells: a new fate for differentiating helper T cells. Immunol Res 2008; 41: 87-102.

21. Langowski JL, Zhang X, Wu L, et al. IL-23 promotes tumour incidence and growth. Nature 2006; 442: 461-5.

22. Lan F, Zhang L, Wu J, Zhang J, Zhang S, Li K, Qi Y, Lin P. IL-23/IL-23R: potential mediator of intestinal tumor progression from adenomatous polyps to colorectal carcinoma. Int I Colorectal Dis 2011; 26: 1511-8.

23. Nguyen GH, Schetter AJ, Chou DB, et al. Inflammatory and microRNA gene expression as prognostic classifier of Barrett's-associated esophageal adenocarcinoma. Clin Cancer Res 2010; 16: 5824-34.

24. Ljujic B, Radosavljevic G, Jovanovic I, et al. Elevated serum level of IL-23 correlates with expression of VEGF in human colorectal carcinoma. Arch Med Res 2010; 41: 182-9. 
25. Adamo V, Franchina T, Minciullo PL, et al. Role of interleukin-23 circulating levels increase in resected colorectal cancer before and after chemotherapy: preliminary data and future perspectives. J Cell Physiol 2011; 226: 3032-4.

26. Stanilov N, Miteva L, Jovchev J, Cirovski G, Stanilova S. The prognostic value of preoperative serum levels of IL-12p40 and IL-23 for survival of patients with colorectal cancer. APMIS 2014; 122: 1223-9.

27. Gangemi S, Minciullo P, Adamo B, et al. Clinical significance of circulating interleukin-23 as a prognostic factor in breast cancer patients. J Cell Biochem 2012; 113: 2122-2125.

\section{Address for correspondence}

\section{Bulent Karagoz}

Department of Medical Oncology

GATA Haydarpasa Hospital

Istanbul, Turkey

e-mail: bulentkaragoz1968@yahoo.com

Submitted: 24.08 .2015

Accepted: 9.03 .2016 